\title{
Methodische Verbesserungen der Phosphat-Bestimmung im Meerwasser mit dem Auto-Analyzer insbesondere für den Bordbetrieb
}

\author{
P. MANGELSDORF \\ Biologische Anstalt Helgoland (Meeresstation); \\ Helgoland, Bundesrepublik Deutschland
}

\begin{abstract}
Methodological improvements of determining phosphate in sea water employing the Auto-Analyzer, especially for shipboard use. A methodological improvement of automated phosphate analysis in sea water is described. The method is based on the photometric measurement of the blue-coloured compound produced by the reaction of phosphate with a single solution reagent containing acid, ammonium molybdate, ascorbic acid, together with a small amount of potassium antimonyl tartrate as a catalyser. The ascorbic acid working solution, as part of the mixing reagent, is made stable for about one week by addition of acetone. The major substances in sea water, especially silicate, do not disturb the analysis. The Auto-Analyzer system was built into a compact unit for easy transport and shipboard use. The reproducibility is approximately $2 \%$ within the range from 0.5 to $8 \mu \mathrm{g}$-at $\mathrm{PO}_{4}-\mathrm{P} / \mathrm{I}$. The method is usable for sea water within a range of salinity from $27 \% 0$ to $36 \%$.
\end{abstract}

\section{EINLEITUNG}

Auf meereskundlichen Forschungsfahrten fallen im Rahmen hydrographischchemischer Untersuchungen in kurzer Zeit große Mengen von Wasserproben an, die entweder adäquat fixiert oder besonders rasch aufgearbeitet werden müssen. In tiefgefrorenem Zustand kann man Wasserproben für die spätere Analyse von z. B. $\mathrm{SiO}_{2}$ und $\mathrm{NO}_{2}-\mathrm{N}$ aufbewahren. Hingegen empfiehlt sich das Tieffrieren nicht für spätere Nitrat-, Ammoniak- und besonders Phosphatanalysen (Grasshorf 1966). Vergleichsmessungen von frischen und tiefgefrorenen Proben ergaben Differenzen im Phosphatgehalt bis zu 50\%. Der Meereschemiker muß daher zahlreiche Analysen dieser wichtigen Minimumstoffe für das Planktonwachstum unmittelbar nach der Probennahme unter den erschwerenden Bordbedingungen durchführen. Daher wird an der Biologischen Anstalt Helgoland an der Verbesserung der Phosphatbestimmung mit Hilfe des Auto-Analyzers gearbeitet, der sich für die im Bordbetrieb besonders vorteilhafte Automatisierung anbietet. Als Grundlagen dienten Arbeiten u. a. von HENRIKSEN (1965), Brewer et al. (1966), Grasshoff (1966) und Chan \& Riley (1966), die alle den Auto-Analyzer für $\mathrm{PO}_{4}$-P-Bestimmungen im Meerwasser eingesetzt haben. Daneben wurde an der Biologischen Anstalt Helgoland zum Vergleich die seit Jahren bewährte 
manuelle Phosphatmessung nach der Methode von Kalle (1934) modifiziert nach NüMANN (1949) angewandt.

\section{ERGEBNISSE}

Einbau des Auto-Analyzersystems in eine kompakte Einheit für den Bordbetrieb

An der Biologischen Anstalt Helgoland wird der Auto-Analyzer an Bord des Forschungskutters „Friedrich Heincke " häufig für kurze Forschungsfahrten eingesetzt. Im Gegensatz zur Verwendung bei großen Forschungsreisen wie z. B. auf der "Meteor" muß man daher das Auto-Analyzersystem schnell und sicher an und von Bord bringen können, möglichst unter Vermeidung fester Einbauten. Es wurde daher folgender Weg

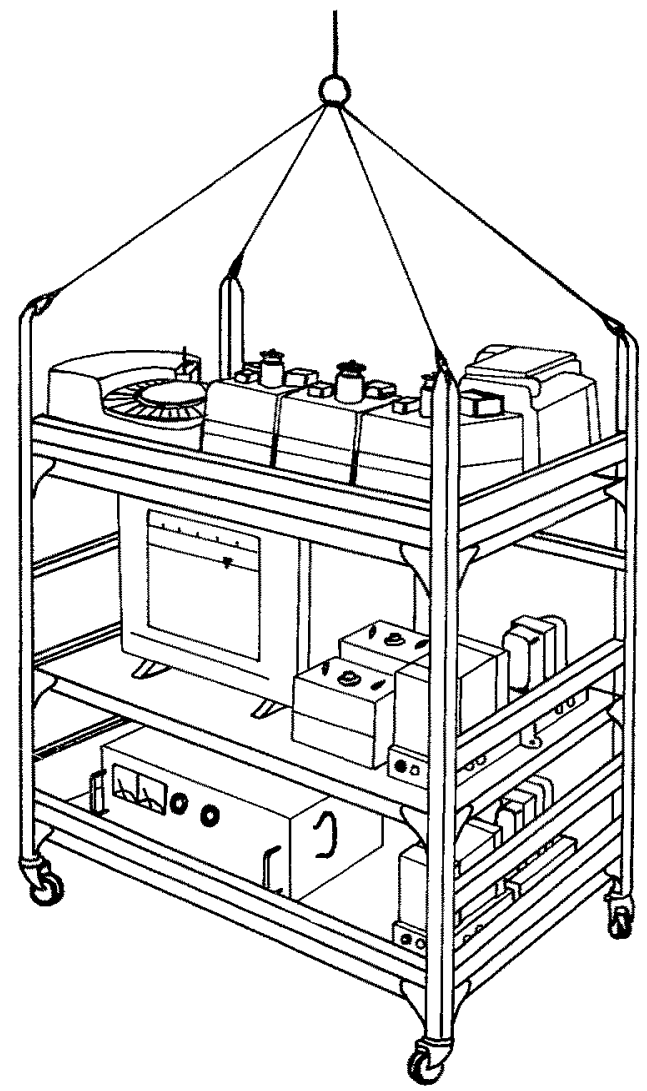

Abb. 1: Das Auto-Analyzersystem als kompakte Einheit für den Betrieb besonders an Bord, mit 3 Kanälen (für $\mathrm{SiO}_{2}, \mathrm{NO}_{2}-\mathrm{N}$ und $\mathrm{PO}_{4}-\mathrm{P}$ ). Obere Etage im Gestell: Probennehmer, Photometer und Proportionierpumpe. Mittlere Etage: Schreiber, Skalenspreizer, Spannungsstabilisator. Untere Etage: Stromversorgung mit Spannungsstabilisator 
beschritten: Das von der Firma TECHNIKON gelieferte System wurde in ein fahrbares Metallgestell mit den Maßen $100 \times 60 \times 110 \mathrm{~cm}$ eingebaut und verbleibt darin für den Labor- und Bordbetrieb (Abb. 1). Ein Metallrahmen mit mehreren Etagen nimmt alle Teile des Systems wie Probennehmer, Pumpe, Verweilspiralen, Photometer und Schreiber sowie die Stromversorgung auf. Diese kompakte Einheit ist leicht von Land mit dem bordeigenen Ladegeschirr an Deck zu setzen. Der Auto-Analyzer hat sich in jahrelangem Betrieb unter rauhen Bedingungen auf dem Forschungskutter „Friedrich Heincke" gut bewährt. Die Gummilagerung der Photometer verhindert ein Ubertragen der Schiffsmaschinenvibration. Die feststellbaren Gummiräder am Metallgestell fangen die Erschütterung durch das Stampfen des Schiffes weitgehend auf.

\section{Verbesserungen der automatischen Phosphatbestimmung}

Der automatischen Bestimmung des gelösten anorganischen Phosphats im Meerwasser, die auf der Methode von MuRPhy \& RILEY (1962) modifiziert nach Grasshoff (1970) basiert, liegt nach Grasshorf folgende chemische Reaktion zugrunde: Das im Meerwasser vorhandene Orthophosphat bildet im pH-Bereich von 1,5 zusammen mit Molekülen der Hexamolybdänsäure Molybdatophosphorsäure. Diese Reaktion wird durch Antimonyltartrat katalytisch beschleunigt. Die Ascorbinsäure als Teil des Mischreagenz reduziert die Heteropolysäure um zwei Aquivalente. Die blaue Reduktionsverbindung, deren Entstehung im einzelnen noch nicht geklärt ist, bleibt bei konstanten Arbeitsbedingungen stabil. Die Reaktionszeit beträgt bei der manuellen Methode 30 Minuten. Sie richtet sich bei bisher bekannten Phosphatanalysen der genannten Autoren mit dem Auto-Analyzer nach den unterschiedlichen Fließsystemen und liegt in der Zeit von 10 bis 20 Minuten. Bei unseren Arbeiten mit der automatischen Phosphatanalyse nach Bernhard \& MACchi (1965), ARMStrong et al. (1966), Brewer et al. (1966), Grasshoff (1966), Chan \& Riley (1966) und Grasshoff (1970) stellten sich einige Punkte als nachteilig heraus, die wir zu verbessern versucht haben: Entgegen den angegebenen Analysenvorschriften mußte die Ascorbinsäure als Teil des Mischreagenz zum Verbrauch täglich neu angesetzt werden, während sich die Stammlösung nur bei etwa $1-3^{\circ} \mathrm{C}$ relativ lange stabil hält. Die Ascorbinsäure wurde von uns daher durch Zugabe von Aceton stabilisiert. Die Haltbarkeit der Arbeitslösung beträgt dann etwa eine Woche.

Zur Beschleunigung der Reaktion wurden allgemein erhöhte Temperaturen empfohlen (bis $70^{\circ} \mathrm{C}$ ). Dabei stört jedoch nach Grasshoff (1966) das Silikat durch Eigenfärbung bzw. Ausfällung erheblich. Wir fanden, daß eine Temperaturerhöhung auf $39^{\circ} \mathrm{C}$ keine Störung durch das Silikat im Meerwasser verursachte. Gleichzeitig wurde der $\mathrm{pH}$-Wert der Ascorbinsäure unter Verwendung von Levor IV (TECHNIKoN Reagenz) und Verdünnung der Konzentration auf $\mathrm{pH} 1,0$ herabgesetzt. Die in der Literatur angegebenen Schlauchsysteme hatten einen relativ großen Durchmesser, weswegen die Probenfrequenz zu klein wurde. Das Verhältnis Probe zu Sperflüssigkeit erwies sich als ungünstig. Bei zu kurzer Spülzeit der Sperrflüssigkeit zeichnet der Schreiber keine saubere Basislinie zwischen den Peaks auf, da das Schlauchsystem dann nicht genügend gespült werden kann. Die Folge ist ein Addieren des „Peakrestes“ zur nächsten 
Probe. Dieser Meßwert wird dann bei gleichem Phosphatgehalt zu hoch ausfallen. Nach mündlicher Mitteilung von BERnHARd (1969) stellt die Vergrößerung des Schlauchsystems zwar eine Lösung zur besseren Ausprägung der Peaks auf dem Schreiber dar, allerdings schien uns die damit verbundene Verringerung der Probenfrequenz angesichts der großen Probenzahl bei Forschungsfahrten an Bord wenig wünschenswert.

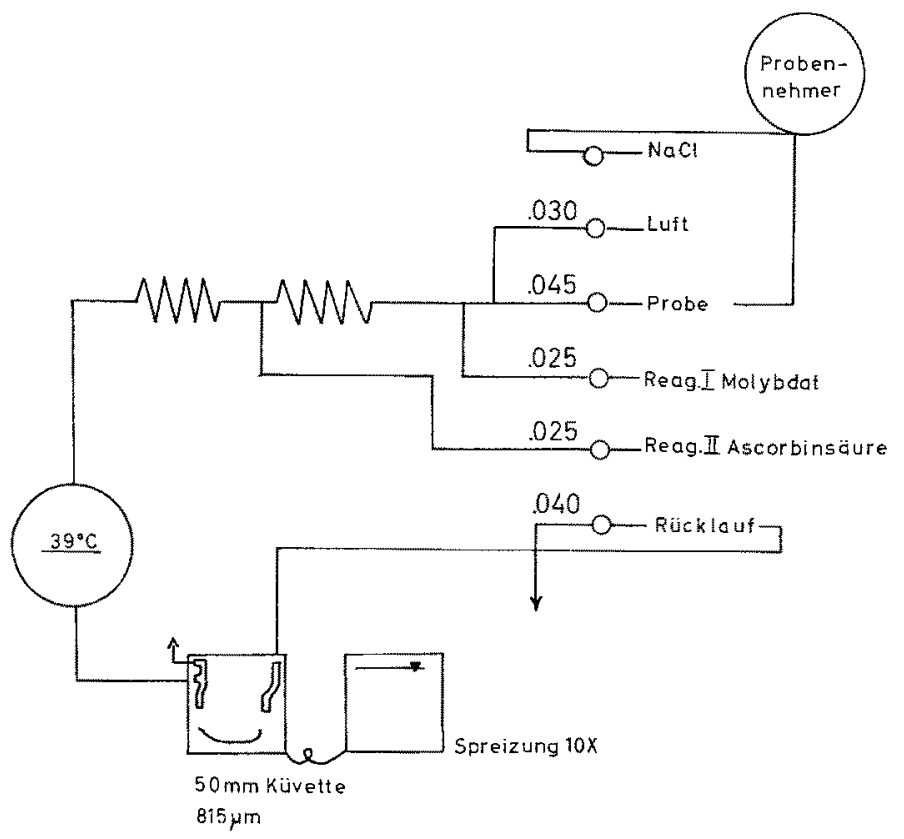

Abb. 2: Fließdiagramm für den Auto-Analyzer zur automatischen Analyse von anorganisch gelöstem Phosphat im Meerwasser

Wir verwendeten daher das in Abbildung 2 skizzierte Schlauchsystem mit geringeren Volumina. Daraus resultiert ein günstiges Verhältnis Probe zu Sperrflüssigkeir, was eine Erhöhung der Probenfrequenz ermöglicht. Die Frequenz von 20 Proben pro Stunde stellt einen Kompromiß zwischen gew ünschter rascher Analysenfolge und sauberer Trennung der Peaks dar.

Auf Forschungsfahrten reicht diese Analysenfrequenz aus, da hiermit 30 Minuten nach der Entnahme von sieben Wasserproben aus den Schöpfern die Meßwerte (drei Kanäle: $\mathrm{SiO}_{2}, \mathrm{NO}_{3}-\mathrm{N}, \mathrm{PO}_{4}-\mathrm{P}$ ) vorlagen. Sie konnten dann bereits bei der nächsten Station für eine gezielte Probennahme in Rechnung gestellt werden.

Die Sperrflüssigkeit zur Probentrennung und Spülung des Systems besteht aus in destilliertem Wasser gelöstem NaCl. Wir prüften eine Sperrflüssigkeit von Salzgehalten mit $28 \%$ bis $40 \%$ in Stufen von $1 \%$. Wie sich zeigte, hatten Veränderungen im Salzgehalt keinen Einfluß auf die Spülung des Systems bzw. auf den Rückgang des Schreibers zur Basislinie. Während GRASsHoff (1970) künstliches Seewasser empfiehlt, verwendeten wir für alle Meerwasser eine Sperrflüssigkeit von $\mathrm{NaCl} 32 \%$. 
Um den Einfluß des Salzgehaltes der Proben auf die Phosphatanalyse zu untersuchen, wurden Eichreihen mit künstlichem Seewasser verschiedenen Salzgehalts analysiert, und zwar von $20 \%, 25 \%, 28 \%, 30 \%, 32 \%, 34 \%, 36 \%$ und $37,5 \%$. Es zeigte sich, daß der Salzgehalt erheblich variieren kann, ohne daß dies Rückwirkungen auf den Meßwert hat. Dieses Ergebnis erwies sich als günstig, da Analysen von Meerwasser mit 27 bis $35 \%$ Salzgehalt regelmäßig anfallen.

Das Berechnen jedes Einzelpeaks von der Basislinie am Schreiber aus wurde durch die Herstellung eines Ableselineals erleichtert, das ein direktes Ablesen der Konzentration in $\mu \mathrm{g}$-at $\mathrm{PO}_{4}-\mathrm{P} / 1$ gestattet. Im Gegensatz zur manuellen Vergleichsmessung ist die Beziehung von Konzentration zur Peakhöhe beim Auto-Analyzer nicht linear.

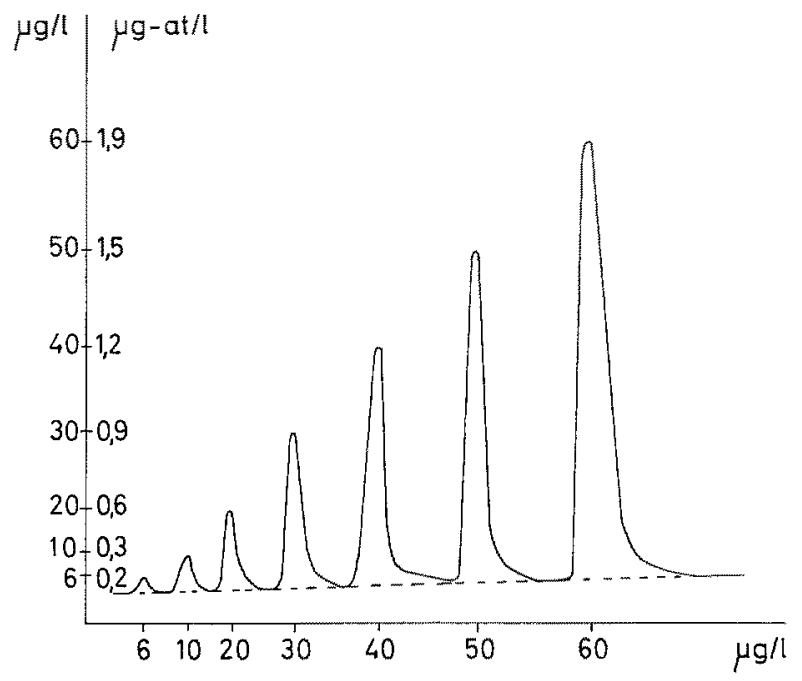

Abb. 3: Registrierung von Werten einer Eichreihe von Phosphat-Phosphor in künstlichem Meerwasser

Gegenüber anderen Angaben wurde eine 10fache Skalenspreizung am Schreiber eingeführt, da Peaks unter $0,5 \mu \mathrm{g}$-at $\mathrm{PO}_{4}-\mathrm{P} / 1$ sich sonst kaum aus der Basislinie hervorheben. In Abbildung 3 sind die Peaks einer Eichreihe mit zehnfacher Spreizung aufgetragen. Zur Ermittlung der Basislinie am Schreiber wurde nach 30 Proben für etwa 5 Minuten für den Reagenzblindwert die Reagenzbasislinie ermittelt, an der das $\mathrm{Ab}-$ leselineal angelegt wurde.

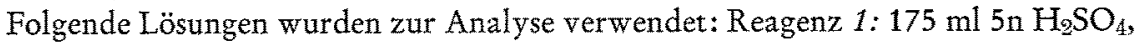
$53 \mathrm{ml} 4 \%$ ige Ammoniumheptamolybdatlösung und $18 \mathrm{ml}$ Kaliumantimonyltartrat $(0,274 \mathrm{~g} / 100 \mathrm{ml}$ destilliertem Wasser) werden in $500 \mathrm{ml}$ destilliertem Wasser gelöst; die Lösung ist kühl und dunkel aufzubewahren. Reagenz 2: 2,2 g Ascorbinsäure werden in $250 \mathrm{ml}$ destilliertem Wasser gelöst. Zugabe von $15 \mathrm{ml}$ Aceton p. a. $+2 \mathrm{ml}$ Levor IV (Technikon Reagenz). Reagenz 1 ist etwa ein Monat haltbar, nach zu langer Lagerung entsteht ein Farbton, der sich nachteilig auf die Analyse auswirkt. 
Vergleichsmessungen mit dermanuellen Methode

Es wurden Vergleichsmessungen des $\mathrm{PO}_{4}-\mathrm{P}$ Gehalts mit der beschriebenen automatischen und der konventionellen manuellen Methode durchgeführt. Dazu wurden zahlreiche Proben von der "Helgoland-Reede" sowie Proben relativ sauberen Meerwassers aus dem Golf von Biscaya verwendet (Tab. 1). Die Streuung und Reproduzierbarkeit der automatischen Methode liegen bei $3 \%$ im untersuchten Bereich yon 0,5

\section{Tabelle 1}

Vergleichsmessungen des Phosphats $\left(\mu \mathrm{g}\right.$-at $\left.\mathrm{PO}_{4}-\mathrm{P} / 1\right)$ der automatischen Methode (Auto-Analyzer) und der manuellen Methode nach Kalle (1934) modifiziert nach NüManN (1949). Die Zahlen in Klammern repräsentieren Mittelwerte von je 10 Analysen, die übrigen Zahlen sind Einzelmessungen

Nordseewasser: Proben von der Station "Helgoland Reede" (Salzgehalt 32,5-33,5\%)

Ozeanwasser: Proben aus der Biscaya

Manuelle
Bestimmung

Auto-Analyzer

(Salzgehalt 35,0-35,5\%)

\begin{tabular}{cccc} 
Bestimmung & Auto-Analyzer & Bestimmung & Auto-Analyzer \\
\hline 0,10 & & 0,11 & 0,10 \\
0,22 & 0,11 & 0,17 & 0,17 \\
0,26 & 0,22 & 0,23 & 0,22 \\
0,28 & 0,25 & 0,26 & 0,25 \\
0,29 & 0,28 & 0,34 & 0,35 \\
0,36 & 0,30 & 0,37 & 0,36 \\
0,40 & 0,34 & 0,41 & 0,40 \\
0,48 & 0,34 \\
0,50 & 0,47 & 0,44 & 0,44 \\
0,57 & 0,50 & 0,46 & 0,47 \\
0,64 & 0,55 & 0,49 & 0,51 \\
1,01 & 0,60 & 0,55 & 0,55 \\
1,33 & 1,02 & 0,58 & 0,60 \\
1,95 & 1,32 & 0,59 & 0,61 \\
2,04 & 1,99 & 0,66 & 0,67 \\
5,02 & 2,10 & 0,67 & 0,67 \\
6,54 & 5,28 & 0,70 & 0,69 \\
& 6,81 & 0,71 & 0,73 \\
\end{tabular}

bis $8 \mu$ g-at $\mathrm{PO}_{4}-\mathrm{P} / \mathrm{l}$. Meßwerte von der "Helgoland-Reede" lagen im Bereich von 0,6-4 $\mu$ g-at $\mathrm{PO}_{4}-\mathrm{P} / \mathrm{l}$, Werte aus der Biscaya $z$ wischen $0,3-0,7 \mu \mathrm{g}$-at $\mathrm{PO}_{4}$-P/l. Es zeigte sich, daß bei Mengen unter $0,1 \mu \mathrm{g}$-at $\mathrm{PO}_{4}-\mathrm{P} / 1$ die Differenz zur manuellen Methode auf $3-4 \%$ anstieg. $\mathrm{Ab} 10 \mu \mathrm{g}$-at $\mathrm{PO}_{4}-\mathrm{P} / 1$ weichen die automatisch aufgezeichneten Ergebnisse erheblich von denen der manuellen Methode ab (bis zu $5 \%$ ).

\section{ZUSAMMENFASSUNG}

1. Es werden methodische Verbesserungen der automatischen Messung von gelöstem anorganischen Phosphat im Meerwasser mit Hilfe des Auto-Analyzers beschrieben (MURPHY \& RILEY 1962, modifiziert nach Grasshoff 1965). Die Verbesserungen 
machen diese Methode für den Bordbetrieb auf Forschungsschiffen besonders geeignet, zumal das Auto-Analyzersystem als kompakte Einheit zum besseren Transport auf das Schiff in ein Gestell eingebaut wurde. Die Reagenzien werden abgewogen an Bord mitgeführt. Das Arbeitsreagenz Ascorbinsäure als Teil des Mischreagenzes konnte für eine Woche bei Arbeitstemperatur haltbar gemacht werden.

2. Die Reaktion wird durch die wichtigsten im Meerwasser vorhandenen Substanzen, insbesondere Silikat, nicht beeinflußt. Streuung und Reproduzierbarkeit liegen bei $3 \%$ im Bereich von 0,5-8 $\mu$ g-at $\mathrm{PO}_{4}$-P/1. Die Methode ist für Meerwasser mit einem Salzgehalt von $27 \%$ bis $35 \%$ geeignet.

Danksagung. Herm Dr. W. Hickel (Biologische Anstalt Helgoland) danke ich für die Kritik und eingehende Hilfe bei der Zusammenstellung und Gliederung des Manuskriptes. Herrn R. BENESCH und Herrn E. H. HaRms (Biologische Anstalt Helgoland) danke ich für wertvolle Hinweise. Frl. I. Jokele und Frl. S. Ohlsen gilt mein Dank für die Anfertigung der Abbildungen.

\section{ZITIERTE LITERATUR}

Armstrong, F. A. J., Stearns, C. R. \& Strichland, J. D. H., 1967. The measurement of upwelling and subsequent biological processes by means of the Technicon Autoanalyzer and associated equipment. Deep Sea Res. 14, 381-389.

Bernhard, M. \& Macchi, G., 1965. Anwendung und Möglichkeiten der automatischen chemischen Analyse mit dem AutoAnalyzer in der Ozeanographie. Automation in der Analytischen Chemie. Technicon Symp., 83-93.

BreWer, P. G., ChaN, K. M. \& RuLEY, J. P., 1966. Automatic determination of certain micronutrients in sea water. Automation in Analytical Chemistry, Technikon Symp. 5.

CHAN, K. M. \& RuEx, J. P., 1966. The automatic determination of phosphate in sea water. Deep Sea Res. 13, 467-471.

GrasshofF, K., 1966. Uber automatische Methoden zur Bestimmung von Fluorid, gelöstem Phosphat und Silikat in Meerwasser. Kieler Meeresforsch, 22, 42-46.

- 1970. A simultaneous multiple channel system for nutrient analysis in seawater with analog and digital data record. Technicon Corporation $R$ 893, 133-145.

HenRtKSEn, A., 1965. An automatic method for determining low-level concentrations of phosphates in fresh and saline waters. Analyst, Lond. 90, 29-34.

- 1966. Interference from silica in phosphate analysis. Analyst, Lond. 91, 290-291.

KALLE, K., 1934. Meereskundliche chemische Untersuchungen mit Hilfe des Zeißschen Pulfrich Photometers. 3. Mitt. Methode Untersuchung der Phosphatgehaltsbestimmung. Annln Hydrogr., Berl. 62, 65-74.

Murphy, J. \& Riley, J. P., 1962. A modified single solution method for the determination of phosphate in natural waters. Analytica dim. Acta 27, 31-36.

NüManN, W., 1949. Kolorimetrische Methoden zur quantitativen Bestimmung von Silikat und organisch wie anorganisch gebundenem Phosphor und Stickstoff im Meerwasser unter Benutzung des Pulfrich Photometers. Dt. hydrogr, Z. 2, 137-153.

Anschrift des Autors: P. MangelsdorF

Biologische Anstalt Helgoland (Meeresstation)

2192 Helgoland

Bundesrepublik Deutschland 